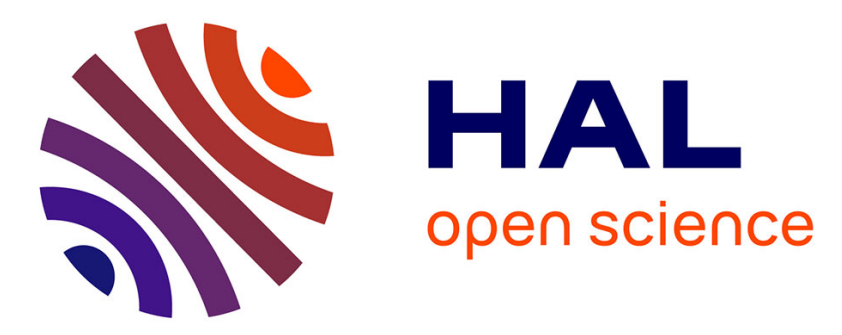

\title{
On-line parameter estimation via algebraic method: An experimental illustration
}

Romain Delpoux, Thierry Floquet

\section{To cite this version:}

Romain Delpoux, Thierry Floquet. On-line parameter estimation via algebraic method: An experimental illustration. Asian Journal of Control, 2015, 17 (1), pp.315-326. 10.1002/asjc.870 . hal00933292

\section{HAL Id: hal-00933292 \\ https://hal.inria.fr/hal-00933292}

Submitted on 11 Mar 2014

HAL is a multi-disciplinary open access archive for the deposit and dissemination of scientific research documents, whether they are published or not. The documents may come from teaching and research institutions in France or abroad, or from public or private research centers.
L'archive ouverte pluridisciplinaire HAL, est destinée au dépôt et à la diffusion de documents scientifiques de niveau recherche, publiés ou non, émanant des établissements d'enseignement et de recherche français ou étrangers, des laboratoires publics ou privés. 


\title{
On-line parameter estimation via algebraic method: An experimental illustration.
}

\author{
R. Delpoux and T. Floquet
}

\begin{abstract}
This article presents an algebraic on-line parameters estimation method for Linear Time Invariant (LTI) systems subject to polynomial perturbations. Particular attention is given to practical implementation. The estimator may be expressed as simple FIR filter, which is advantageous for online application, since the coefficients may be computed offline. The robustification against possible singularities is shown and the approach has the advantage of eliminating singularities that may occur in the experimental identification. This algorithm is illustrated experimentally on a Permanent Magnet Stepper Motor (PMSM) and a magnetic bearing for which parametric estimation is known to be a difficult task. The parameters to be estimated are sometimes measurable with difficulties and may slightly vary over time.
\end{abstract}

Key Words: Parameter identification, Algebraic method, Magnetic bearing, PMSM.

\section{INTRODUCTION}

This article is concerned with parameter identification based on an algebraic method. The general framework of this method has been introduced by M. Fliess and H. Sira-Ramirez [13, 14]. It is based on differential algebra and operational calculus. Unlike traditional methods, the proposed estimator is non-asymptotic: the solutions are obtained by exact algebraic formulas, one to obtain estimates in finite time. The desired parameters are expressed as a function of integrals of the measured outputs and inputs of the system. It does not need any statistical knowledge of the noise (for instance, the assumption that the noise is Gaussian is not required). Moreover, the estimator is able to treat identification in the presence of bias and of structured perturbations ( $c f$. Definition 4 of [21]). For these reasons, the algebraic method has been applied to many fields such as abrupt change detection, delay identification [3, 4, 12, 31], and numerical differentiation $[22,26]$.

Many different methods for parameter identification exist in the literature. One of the most popular concepts is the regression (linear or nonlinear), such as in [29,6]. Observer based approaches can also be found, such as asymptotic observers using the extended Kalman filter [5] or finite time ones like sliding modes observers $[1,2,15,25]$. The general framework of parameter identification via algebraic method initially was proposed in [21], based on Mikusiński's field of operators. The theory and the methodology are described and illustrated with

R. Delpoux is with LAGIS (CNRS UMR 8219) \& Inria Non-A Project, Université Lille 1, France, roma in. delpoux@ inria.fr and T. Floquet is with LAGIS (CNRS UMR 8219) \& Inria Non-A Project, École Centrale de Lille, France, thierry.floquet dec-lille.fr.

This work was supported by EU Interreg IV A 2 Mers Seas Zeeen Cross- border Cooperation Programme under SYSIASS project 06-020. It was also supported by Nord-Pas de Calais Regional Council and FEDER through the Contrat de Projets Etat Region (CPER) 2007-2013.

The authors would like to thank Laurentiu Hetel for his constructive comments on the article. 
numerical simulation. In [23], an experimental application was proposed for a one direction magnetic levitation system for the identification of one parameter with the annihilation of a constant perturbation. The identification of several parameters has experimentally been applied for a LTI system in [9]. In the literature, one can find articles dealing with estimation for sinusoidal signal parameters [32, 33]. Linear infinite dimensional systems parameter identification was proposed in [27] and the approach was developed for delay systems and systems described by spatially-dependent partial differential equations (PDEs).

This paper presents a method for parameter identification in the case of $n^{t h}$ order LTI systems subject to polynomial perturbations. While the general framework was introduced in [21], in this paper, particular attention is given to the practical implementation. Explicit formulas are given for the integral computations. The expressions are written as FIR filters, which allows their coefficients to be computed offline and provides more efficient estimators for real time application. Finally, an approach is proposed to robustify the estimation. The approach has the advantage of eliminating singularities that may occur in the experimental identification. Finally a concrete application of the results is applied to two different electromechanical systems: (i) a Permanent Magnet Stepper Motor (PMSM) and (ii) a magnetic bearing. Both systems are nonlinear, and several parameters have to be identified simultaneously.

This article is divided into three parts. Section II presents a general formula for the estimation of the parameters of LTI systems subject to perturbations. In the second section, practical issues are introduced concerning the implementation of the estimator. The last section is devoted to the identification of the parameters on two experimental applications where the performance of the algorithm is highlighted.

\section{Algebraic Approach}

The parameter identification using an algebraic approach for linear time invariant differential equations has been studied in several papers. The obtained analytical expressions are not unique. Different expressions can be derived $[16,22,27,30,32,18]$. Consider the following LTI system subject to perturbations:

$$
\sum_{i=0}^{n} a_{i} y^{(i)}(t)=\sum_{i=0}^{m} b_{i} z^{(i)}(t)+\varpi(t) .
$$

where the $a_{i}$ and $b_{i}$ are the system parameters (to be identified), with $a_{n}=1$ and $m<n . y(t) \in \mathbb{R}$ and $z(t) \in \mathbb{R}$ are measured quantities of the system. The variable $\varpi(t)=\sum_{i=1}^{\kappa} c_{i} \frac{t^{i-1}}{(i-1) !}, \kappa \in \mathbb{N}$ stands for perturbations or uncertainties assumed to be polynomial.

Theorem 1 An estimation of the unknown parameters $a_{0}, \ldots, a_{n-1}, b_{0}, \ldots b_{m}$ of equation (1) is given by:

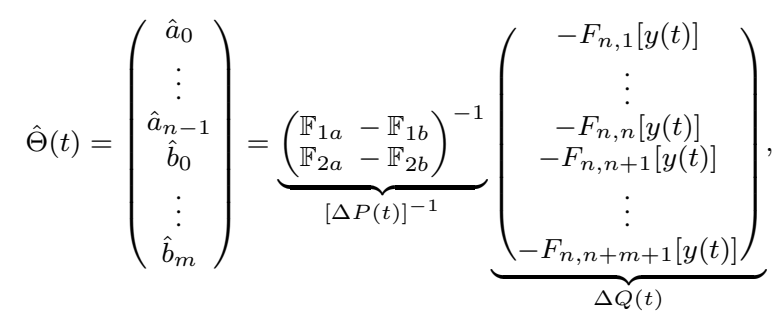

with:

$\mathbb{F}_{1 a}=\left(\begin{array}{ccc}F_{0,1}[y(t)] & \ldots & F_{n-1,1}[y(t)] \\ \vdots & \vdots & \vdots \\ F_{0, n}[y(t)] & \ldots & F_{n-1, n}[y(t)]\end{array}\right)$,
$\mathbb{F}_{1 b}=\left(\begin{array}{ccc}F_{0,1}[z(t)] & \ldots & F_{m, 1}[z(t)] \\ \vdots & \vdots & \vdots \\ F_{0, n}[z(t)] & \ldots & F_{m, n}[z(t)]\end{array}\right)$,
$\mathbb{F}_{2 a}=\left(\begin{array}{ccc}F_{0, n+1}[y(t)] & \ldots & F_{n-1, n+1}[y(t)] \\ \vdots & & \vdots \\ F_{0, n+m, 1}[y(t)] & \ldots & F_{n-1, n+m+1}[y(t)]\end{array}\right)$, 
$\mathbb{F}_{2 b}=\left(\begin{array}{ccc}F_{0, n+1}[z(t)] & \ldots & F_{m, n+1}[z(t)] \\ \vdots & \vdots & \vdots \\ F_{0, n+m+1}[z(t)] & \ldots & F_{m, n+m+1}[z(t)]\end{array}\right)$,

where

$$
F_{i, p}[f(t)]=\sum_{j=n-i}^{\kappa+n} \frac{c_{i, j} \int_{0}^{t}(t-\tau)^{\gamma}(-\tau)^{j} f(\tau) d \tau}{\gamma !},
$$

with

$$
\begin{aligned}
\gamma & =\kappa+2 n+p-i-j-1, \\
c_{i, j} & =\left(\begin{array}{c}
\kappa+n \\
j
\end{array}\right) \frac{(\kappa+i) !}{(i+j-n) !},
\end{aligned}
$$

and $p \in\{1, \ldots, n+m+1\}$ represents the number of unknown parameters to be estimated.

Proof of Theorem 1: The expressions obtained in Theorem 1 are constructed as follows:

Consider the Laplace transformation of (1):

$$
\begin{aligned}
& \sum_{i=0}^{n} a_{i}\left(s^{i} Y(s)-s^{i-1} y(0)-\ldots-y^{(i-1)}(0)\right) \\
& \quad=\sum_{i=0}^{m}\left(s^{i} Z(s)-s^{i-1} z(0)-\ldots-z^{(i-1)(0)}\right. \\
& \quad+\sum_{i=0}^{\kappa} \frac{c_{i}}{s^{i}}
\end{aligned}
$$

The initial conditions, as well as the perturbations are considered as undesired parameters. The perturbations, being structured, are easily annihilated by multiplying both sides of (4) with $s^{\kappa}$, followed by a derivation of order $\kappa$. The initial conditions are eliminated by differentiating equation (4) $n$ times more . It leads to the linear differential operator:

$$
\Pi=\frac{d^{\kappa+n}}{d s^{\kappa+n}} s^{\kappa} .
$$

In light of the Leibniz formula:

$$
\frac{d^{h}(X(s) Y(s))}{d s^{h}}=\sum_{j=0}^{h}\left(\begin{array}{c}
h \\
j
\end{array}\right) \frac{d^{h-j}(X(s))}{d s^{h-j}} \frac{d^{j}(Y(s))}{d s^{j}},
$$

and the relationship:

$$
\frac{d^{k}\left(s^{l}\right)}{d s^{k}}= \begin{cases}\frac{l !}{(l-k) !} s^{l-k}, & \text { if } 0<k \leq l \\ 0, & \text { if } 0<l<k \\ \frac{(-1)^{k}(k-l-1) !}{(-l-1) !} s^{l-k}, & \text { if } l<0<k\end{cases}
$$

one obtains:

$$
\begin{aligned}
& \sum_{i=0}^{n} a_{i}\left(\sum_{j=n-i}^{\kappa+n}\left(\begin{array}{c}
\kappa+n \\
j
\end{array}\right) \frac{(\kappa+i) ! s^{i+j-n}}{(i+j-n) !} \frac{d^{j}(Y(s))}{d s^{j}}\right) \\
& =\sum_{i=0}^{m} b_{i}\left(\sum_{j=n-i}^{\kappa+n}\left(\begin{array}{c}
\kappa+n \\
j
\end{array}\right) \frac{(\kappa+i) ! s^{i+j-n}}{(i+j-n) !} \frac{d^{j}(Z(s))}{d s^{j}}\right) .
\end{aligned}
$$

Recall that derivation w.r.t. $s$ in the operational domain translates into multiplication by $-t$ in the time domain. Multiplication by $s$ in the operational domain corresponds to derivation in the time domain. Applying the linear estimator (8) is not appropriate. Derivation amplifies the high frequency components and consequently, the noise 
contribution. A simple solution is to make the estimator proper. It is enough to multiply both sides of (8) by $s^{-(\kappa+n+p)}$ to eliminate the derivation terms and obtain a relationship in the function of integral operators:

$$
\begin{aligned}
& \sum_{i=0}^{n} a_{i}\left(\sum_{j=n-i}^{\kappa+n} \frac{c_{i, j}}{s^{\kappa+2 n+p-i-j}} \frac{d^{j}(Y(s))}{d s^{j}}\right) \\
& =\sum_{i=0}^{m} b_{i}\left(\sum_{j=n-i}^{\kappa+n} \frac{c_{i, j}}{s^{\kappa+2 n+p-i-j}} \frac{d^{j}(Z(s)}{d s^{j}}\right),
\end{aligned}
$$

where:

$$
c_{i, j}=\left(\begin{array}{c}
\kappa+n \\
j
\end{array}\right) \frac{(\kappa+i) !}{(i+j-n) !} .
$$

Use the following formulas to obtain (9) in the time domain:

$$
\mathscr{L}^{-1}\left(\frac{1}{s^{l}} \frac{d^{k} Y(s)}{d s^{k}}\right)=\int_{0}^{t} \ldots \int_{0}^{\tau_{2}}\left(-\tau_{1}\right)^{k} y\left(\tau_{1}\right) d \tau_{1} \ldots d \tau_{l},
$$

for $l \geq 1$, and:

$$
\int_{0}^{t} \ldots \int_{0}^{\tau_{2}} y\left(\tau_{1}\right) d \tau_{1} \ldots d \tau_{l}=\int_{0}^{t} \frac{(t-\tau)^{l-1} y(\tau)}{(l-1) !} d \tau
$$

These two formulas together give:

$$
\mathscr{L}^{-1}\left(\frac{1}{s^{l}} \frac{d^{k} Y(s)}{d s^{k}}\right)=\int_{0}^{t} \frac{(t-\tau)^{l-1}(-\tau)^{k} y(\tau)}{(l-1) !} d \tau .
$$

In the time domain, equation (9) leads to:

$$
\begin{aligned}
& \sum_{i=0}^{n} a_{i}\left(\sum_{j=n-i}^{\kappa+n} \frac{c_{i, j} \int_{0}^{t}(t-\tau)^{\kappa+2 n+p-i-j-1}(-\tau)^{j} y(\tau) d \tau}{(\kappa+2 n+p-i-j-1) !}\right) \\
& =\sum_{i=0}^{m} b_{i}\left(\sum_{j=n-i}^{\kappa+n} \frac{c_{i, j} \int_{0}^{t}(t-\tau)^{\kappa+2 n+p-i-j-1}(-\tau)^{j} z(\tau) d \tau}{(\kappa+2 n+p-i-j-1) !}\right) .
\end{aligned}
$$

with $p \in\{1, \ldots, n+m+1\}$.

One obtains as many equations as parameters to identify. This can be written under the form (2).

Remark 1 Algebraic manipulations to obtain (2) are not unique. A method to find minimal annihilators, as well as differential numerators with good numerical properties once back in the time domain can be found in [33].

\section{Implementation Issues}

In this section, implementation issues are discussed. First, the integrals are rewritten as linear filters in order to optimize the computation time required for on-line estimation. Then, a method is proposed to avoid singularities that may occur when inverting the matrix in (2). 


\subsection{FIR representation of the estimator}

In the previous section, Theorem 1 was proposed to obtain an algebraic estimator for parameter identification. The algebraic estimator is expressed systematically as a combination of expressions similar to (10):

$$
v(t)=\int_{0}^{t} \frac{(t-\tau)^{\gamma}(-\tau)^{k} y(\tau)}{\gamma !} d \tau .
$$

This expression is an integral on the time intervals $[0, t[$. For long time interval, the measurement noise and unmodelled term contributions, such as assumptions on the polynomial perturbation on a window, are considered as unstructured perturbations and have harmful effects on the calculation of integrals. One can mention [19], concerning a study about the noise contribution on these estimators. In order to minimize these effects, the integrals are computed on a sliding window $\left[t-T_{f}, t\right]$, where $T_{f}$ represents the size of the latter .

The integral (12) then is approximated on a sliding horizon by:

$$
\hat{v}(t)=\int_{t-T_{f}}^{t} \frac{(t-\sigma)^{\gamma}\left(t-T_{f}-\sigma\right)^{j}}{\gamma !} f(\sigma) d \sigma
$$

Setting $\sigma_{1}=\tau-\left(t-T_{f}\right)$, we get an expression of integral (13) on the interval $\left[0, T_{f}\right]$ :

$$
\hat{v}(t)=\int_{0}^{T_{f}} \underbrace{\frac{\left(T_{f}-\sigma_{1}\right)^{\gamma}\left(-\sigma_{1}\right)^{j}}{\gamma !}}_{g\left(\sigma_{1}\right)} f\left(t-T_{f}+\sigma_{1}\right) d \sigma_{1} .
$$

It is important to note that the function $g(\sigma)$ is independent of the time $t$. Thus, this function can be computed off-line which reduces the computational load during real-time estimation.

Finally, the time interval is normalized on an interval $\mathcal{I}=[0,1]$ by introducing $\sigma_{2}=\frac{\sigma_{1}}{T_{f}}$. The integral (14) becomes:

$$
\begin{aligned}
& \int_{0}^{1} \underbrace{\frac{T_{f}^{\gamma+j}\left(1-\sigma_{2}\right)^{\gamma}\left(-\sigma_{2}\right)^{j}}{\gamma !}}_{g_{2}\left(\sigma_{2}\right)} f\left(t-T_{f}\left(1-\sigma_{2}\right)\right) d \sigma_{2} \\
& =\int_{0}^{1} g_{2}\left(\sigma_{2}\right) f_{T_{f}}\left(1-\sigma_{2}\right) d \sigma_{2},
\end{aligned}
$$

where $f_{T_{f}}(u)=f\left(t-T_{f} u\right)$. The integral in the interval $[0,1]$ is a classical input-output linear time filtering relation [22].

For implementation purposes, considering that the sampling period $T_{s}$ is constant and that $T_{f}=M T_{s}$, the integrals are approximated with the numerical integration method:

$$
\int_{0}^{1} h(\lambda) d \lambda \approx \sum_{m=0}^{M} W_{m} h\left(t_{m}\right)
$$

where $W_{m}$ and $t_{m}=m T_{s} / T_{f}, m=0, \ldots, M$ are the weights and the abscissas. Using the trapezoidal integration method, the weights correspond to:

$$
W_{0}=W_{M}=\frac{T_{s}}{2} \text { and } W_{m}=T_{s}, m=1, \ldots, M-1 .
$$

From the approximation (16) and from (15), one obtains:

$$
\int_{0}^{1} g\left(\sigma_{2}\right) f_{T_{f}}\left(1-\sigma_{2}\right) d \tau \approx \sum_{m=0}^{M} W_{m} g_{m} f_{M-m},
$$


where $g_{m}=g\left(m T_{s} / T_{f}\right)$ and $f_{M-m}=f_{T_{f}}\left(1-m T_{s} / T_{f}\right)$.

The integral is obtained from the output of a classical FIR digital filter with impulse response $c_{m}=W_{m} g_{m}$, where the input is the sampled signal.

Note that the results obtained from the FIR implementation of the sliding window lead to the implementation using the MAC Unit of a DSP proposed in [16]. The generalized estimator could also be applied to the case of this article.

\subsection{Robust estimation}

From equation (2), the algebraic estimator can be expressed as:

$$
\Delta P \Theta=\Delta Q,
$$

where $\Theta$ is the vector of the parameters to be estimated,

$$
\Delta P=\left(\begin{array}{cc}
\mathbb{F}_{1 a}-\mathbb{F}_{1 b} \\
\mathbb{F}_{2 a}-\mathbb{F}_{2 b}
\end{array}\right) \text { and } \Delta Q=\left(\begin{array}{c}
-F_{n, 1}[y(t)] \\
\vdots \\
-F_{n, n}[y(t)] \\
-F_{n, n+1}[y(t)] \\
\vdots \\
-F_{n, n+m+1}[y(t)]
\end{array}\right)
$$

The vector of estimated parameters is obtained if the matrix $\Delta P$ is invertible. The matrix $\Delta P$ is generically invertible, i.e. for almost every $t$, there are time instants where $\Delta P$ is singular. In order to provide an estimation of the parameters at any instant, one needs to robustify the estimation against possible singularities of the matrix $\Delta P$. Pre-multiply both terms of equation (18) by $(\Delta P)^{T}$. Integrate both terms of the equation during a time interval $\left[t-T_{f}, t\right]$, extracting the constant vector of parameters $\Theta$ out of the integration, to obtain:

$$
S(t) \hat{\Theta}=\left[\int_{t-T_{f}}^{t}(\Delta P)^{T} \Delta Q d \lambda\right],
$$

where $S(t)=\left[\int_{t-T_{f}}^{t}(\Delta P)^{T} \Delta P d \lambda\right]$. This matrix is symmetric and positive definite because $\Delta P$ is generically invertible. For online application and especially when the number of parameters to be estimated is large, solving this linear equation system can be done without computing any determinant, one can use the LU decomposition for example. Since the matrix $S(t)$ is symmetric and positive definite, the Cholesky decomposition would be better, because it is about twice as efficient as the method previously mentioned.

Remark 2 Note that the estimation of the parameters is not obtained before time $t=T_{f}$. Thus, the parameter vector is chosen arbitrarily for $t \in\left[0, T_{f}\left[\right.\right.$. After time $t=T_{f}$, the estimation is obtained from (19).

\section{Applications}

The performance of the estimators is presented experimentally in this section through its application on two different electromechanical processes. The parameter estimation algorithm first is applied for the identification of the electrical parameters of a PMSM. Then, the estimator will be applied on a magnetic bearing which is a more complex example since it is an inherently unstable system where the dynamics are strongly nonlinear. In both cases, the experiments are performed as illustrated in Fig. 1. The value of the parameters to be estimated is chosen arbitrarily at the beginning of the experiment. For a while, the control is applied with the wrong parameters to show the behavior of the misidentified system. Then, at $t=T_{f}$ the parameter estimation starts. Once the time reaches the first sliding window length, the estimated parameters are applied to the control. Choosing the window length is not straightforward because the window should be chosen long enough to attenuate the noise. Indeed, the estimator plays the role of low 


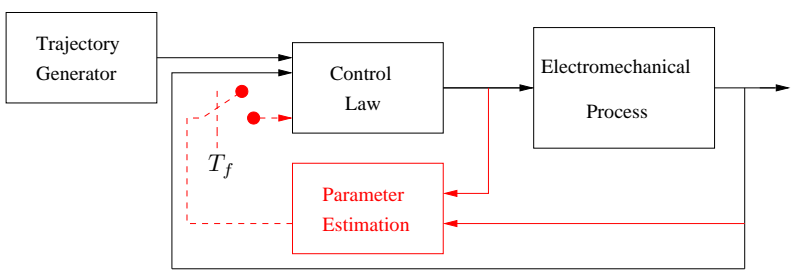

Fig. 1. Estimation procedure.

pass filter. In the absence of perturbations, increasing the window length $T_{f}$ would lead to a more accurate estimation. Nevertheless, the estimator is designed under the assumption that the structured perturbations, to be annihilated, are polynomials on the window. This limits the window length so that the assumption remains true. The computer hardware on the test benches are dSpace cards 1104, with ControlDesk software as interface. Using the library RTlib developed by dSpace, the algorithms are implemented in $\mathrm{C}$ language.

\subsection{Application 1: a PMSM}

\subsubsection{Description of the experiment}

PMSM are widely used in industry for position control, especially in manufacturing applications. PMSM are more robust than brushed DC motors and produce higher torque per volume. They are often controlled in openloop, although the potential loss of synchronism limits operation away from resonances and from high acceleration trajectories. To solve this problem, closed-loop control methods must be used. For such methods to succeed, the model of the motor and its parameters have to be well-known, which brings to the forefront the necessity of parameter identification.

Equations (20) give the standard PMSM model in the phase (or winding) variables:

$$
\left\{\begin{array}{l}
L \frac{d i_{a}}{d t}=v_{a}-R i_{a}+K \omega \sin \left(n_{p} \theta\right), \\
L \frac{d i_{b}}{d t}=v_{b}-R i_{b}-K \omega \cos \left(n_{p} \theta\right), \\
J \frac{d \omega}{d t}=K\left(i_{b} \cos \left(n_{p} \theta\right)-i_{a} \sin (N \theta)\right)-\tau,
\end{array}\right.
$$

where, $v_{a}$ and $v_{b}$ are the voltages applied to the two phases of the PMSM, $i_{a}$ and $i_{b}$ are the two phase currents, $L$ is the inductance of a phase winding, $R$ is the resistance of a phase winding, $K$ is the back-EMF constant (and also the torque constant), $\theta$ is the angular position of the rotor, $\omega=d \theta / d t$ is the angular velocity of the rotor, $n_{p}$ is the number of pole pairs (or rotor teeth), $J$ is the moment of inertia of the rotor (including the load) and $\tau$ represents the load torque.

The phase model can be transformed using Park's transformation [24]. The system (20) is transformed into the so-called $d-q$ model:

$$
\left\{\begin{aligned}
L \frac{d i_{d}}{d t} & =v_{d}-R i_{d}+n_{p} L \omega i_{q}, \\
L \frac{d i_{q}}{d t} & =v_{q}-R i_{q}-n_{p} L \omega i_{d}-K \omega, \\
J \frac{d \omega}{d t} & =K i_{q}-\tau .
\end{aligned}\right.
$$

The $d-q$ transformation is commonly used for PMSM (and synchronous motors in general) because it results in constant voltages and currents at constant speed (instead of the high-frequency phase variables). Also, the model highlights the role of the quadrature current $i_{q}$ in determining the torque.

This application is concerned with the identification of the electrical parameters $R, L$ and $K$. It is important to identify these parameters because they may vary for different reasons, such as heating. Another parameter that could be identified is the inertia $J$. Nevertheless the inertia is identifiable when the acceleration is non-null. In order to identify this parameter, it would be necessary to apply large variations on the motor, so that high acceleration is 
produced. Such trajectories are not suitable for on-line applications. Moreover, this parameter does not really vary and can be estimated off-line using methods similar to those in [10].

With the objective being to identify the electrical parameters, one must consider the electrical equations of (21), and one notes:

$$
\omega_{d}=n_{p} \omega i_{d}, \omega_{q}=n_{p} \omega i_{q}
$$

and

$$
\Gamma_{1}=\frac{1}{L}, \Gamma_{2}=\frac{R}{L}, \Gamma_{3}=\frac{K}{L}
$$

The equations are written under the form:

$$
\left\{\begin{array}{l}
\frac{d i_{d}}{d t}=\Gamma_{1} v_{d}-\Gamma_{2} i_{d}+\omega_{q} \\
\frac{d i_{q}}{d t}=\Gamma_{1} v_{q}-\Gamma_{2} i_{q}-\omega_{d}-\Gamma_{3} \omega .
\end{array}\right.
$$

\subsubsection{Estimation of the electrical parameters}

Several algebraic manipulations can lead to the identification of the three parameters. This identification is performed by generating two equations with the equation of the direct current $i_{d}$ and a third one which is obtained from the equation of the quadrature current $i_{q}$. Thus, we obtain three equations to identify the three parameters $R, L$ and $K$.

In order to apply Theorem 1 , one considers equation (22a) with the quantities $\frac{d i_{d}}{d t}, \omega_{q}, v_{d}$ and $i_{d}$. There is no perturbation in these equations, so $\kappa=0$. Finally, $p=2$ is the number of unknowns. The estimator is expressed as:

$$
\begin{aligned}
& {\left[\begin{array}{ll}
-F_{0,1}\left[v_{d}(t)\right] & F_{0,1}\left[i_{d}(t)\right] \\
-F_{0,2}\left[v_{d}(t)\right] & F_{0,2}\left[i_{d}(t)\right]
\end{array}\right]\left[\begin{array}{l}
\Gamma_{1} \\
\Gamma_{2}
\end{array}\right]=} \\
& {\left[\begin{array}{l}
-F_{1,1}\left[i_{d}(t)\right]+F_{0,1}\left[\omega_{q}(t)\right] \\
-F_{1,2}\left[i_{d}(t)\right]+F_{0,2}\left[\omega_{q}(t)\right]
\end{array}\right],}
\end{aligned}
$$

where, for any function $f(t), F_{i, p}[f(t)]$ are defined in (3).

From the second electrical equation (22b), one can write:

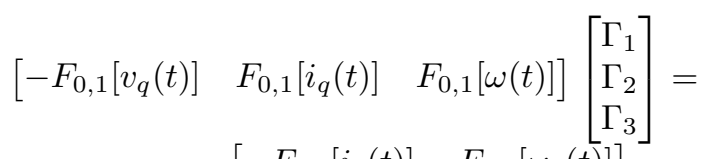

$$
\begin{aligned}
& {\left[-F_{1,1}\left[i_{q}(t)\right]-F_{0,1}\left[\omega_{d}(t)\right]\right] .}
\end{aligned}
$$

Define the vector $\hat{\Gamma}=\left[\begin{array}{lll}\hat{\Gamma}_{1} & \hat{\Gamma}_{2} & \hat{\Gamma}_{3}\end{array}\right]^{T}$. Equations (23) and (24) are rewritten together under the form:

$$
\Delta P \hat{\Gamma}=\Delta Q,
$$

The matrices $\Delta P \in \mathbb{R}^{3 \times 3}$ and $\Delta Q \in \mathbb{R}^{3 \times 1}$ are given by:

$$
\begin{gathered}
\Delta P=\left[\begin{array}{ccc}
-F_{0,1}\left[v_{d}(t)\right] & F_{0,1}\left[i_{d}(t)\right] & 0 \\
-F_{0,2}\left[v_{d}(t)\right] & F_{0,2}\left[i_{d}(t)\right] & 0 \\
-F_{0,1}\left[v_{q}(t)\right] & F_{0,1}\left[i_{q}(t)\right] & F_{0,3}[\omega(t)]
\end{array}\right], \\
\Delta Q=\left[\begin{array}{l}
-F_{1,1}\left[i_{d}(t)\right]+F_{0,1}\left[\omega_{q}(t)\right] \\
-F_{1,2}\left[i_{d}(t)\right]+F_{0,2}\left[\omega_{q}(t)\right] \\
-F_{1,1}\left[i_{q}(t)\right]+F_{1,1}\left[\omega_{d}(t)\right]
\end{array}\right] .
\end{gathered}
$$


One obtains:

$$
\left[\begin{array}{l}
\hat{\Gamma}_{1} \\
\hat{\Gamma}_{2} \\
\hat{\Gamma}_{3}
\end{array}\right]=\Delta P^{-1} \Delta Q .
$$

Finally, the estimated parameters $\hat{L}, \hat{R}$ and $\hat{K}$ are obtained from the relations:

$$
\hat{L}=\frac{1}{\hat{\Gamma_{1}}}, \quad \hat{R}=\frac{\hat{\Gamma_{2}}}{\hat{\Gamma_{1}}} \text { and } \hat{K}=\frac{\hat{\Gamma_{3}}}{\hat{\Gamma_{1}}} .
$$

\subsubsection{Experimental results}

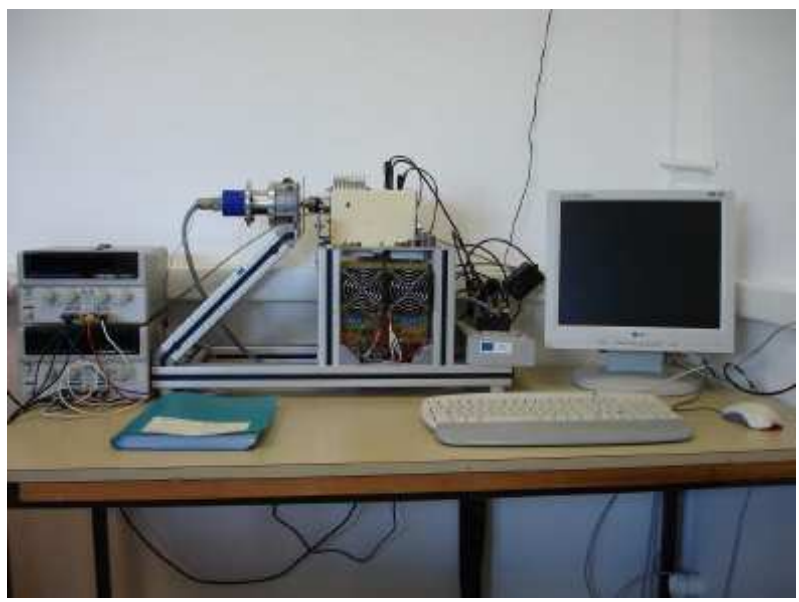

Fig. 2. PMSM test-bench.

The experiments were realized using a stepper motor bench developed in LAGIS at École Centrale de Lille (see Fig. 2). The parameters of the motor with coils in series were identified using the offline procedure described in [10], leading to $L=9 \mathrm{mH}, R=3.01 \Omega, K=0.27 \mathrm{~N} . \mathrm{m} . \mathrm{A}^{-1}$ and $J=3.18 \cdot 10^{-4} \mathrm{~kg} \cdot \mathrm{m}^{2}$. The number of pole pairs is $n_{p}=50$. The input voltages $v_{a}$ and $v_{b}$ of each coil were delivered by two D/A outputs of the dSpace card and amplified by two linear amplifiers. The currents $i_{a}$ and $i_{b}$ are measured using Hall effect sensors with a precision of $1 \%$ of the nominal current $I_{n}=3 \mathrm{~A}$. The power supply provided a maximum voltage $v_{\max }=30 \mathrm{~V}$ and $i_{\max }=3 \mathrm{~A}$. The sampling period for this experiment is constant and equal to $10^{-4} s$ for the control. The results were obtained using a classical velocity tracking control described in [7]. Alternatively, a passivity plus flatness controller [28] or sliding mode controller [8] could have been applied. The reference trajectory was defined such that the parameters were identifiable. Indeed, the parameters are identifiable if the direct current is non-zero. The velocity must also be non-zero in order to identify the back-EMF constant. For this reason, the torque maximization given in [34] was applied, such that at non-zero speed, the direct current was non-zero. The reference velocity was sinusoidal with $\omega^{*}=r \sin (\omega t)+r_{0}$ where $r=5 \mathrm{rad} . \mathrm{s}^{-1}, \omega=5 \cdot 2 \pi \mathrm{rad} . \mathrm{s}^{-1}$ and $r_{0}=20 \mathrm{rad} . \mathrm{s}^{-1}$ was a bias.

The choice of the window length was set experimentally. Experience has shown that a window length $T_{f}=0.2 \mathrm{~s}$ gives good results. With a sampling period of $1.10^{-4} s$, it gives a window of 2000 points. Such a long window is not computable online. To solve this problem, the estimation was sampled at $10^{-3} \mathrm{~s}$, leading to a window length of 200 points. At the beginning of the experiment, the three parameters to be estimated are chosen arbitrarily: $R=5 \Omega$, $L=0.03 H$ and $K=0.4 N . m . A^{-1}$. As this system is stable by nature, the arbitrary parameters can be chosen far from the true parameters.

Fig. 3 shows the identification of the parameters $R, L$ and $K$. The black line represents the instant $\tau$ where the estimation started. At $t=\tau+T_{f}$, with $T_{f}=0.2 s$, the estimated parameters were applied to the control. The parameters $\hat{R}=3.1 \Omega, \hat{L}=9.7 \mathrm{mH}$ and $\hat{K}=0.28 \mathrm{~N} . \mathrm{m} . A^{-1}$ were obtained, as compared with $L=9 \mathrm{mH}, R=3.01 \Omega$ and $K=0.27 N . m . A^{-1}$. 

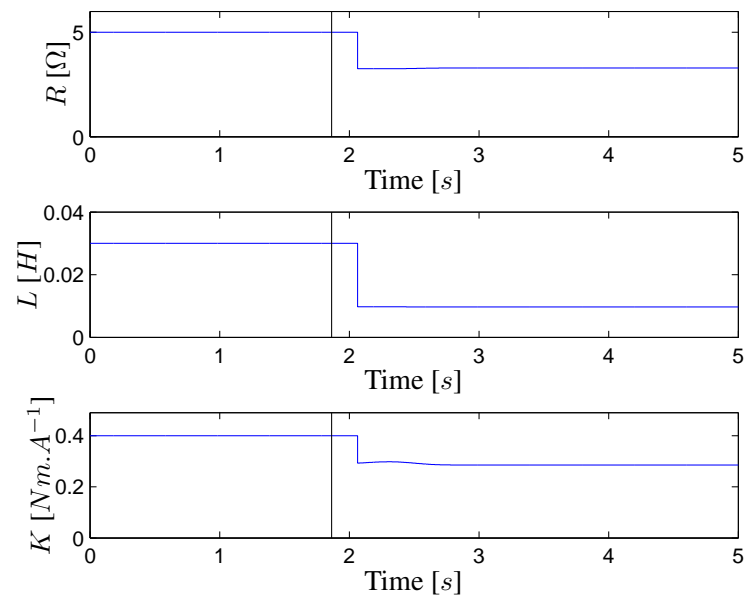

Fig. 3. Estimation of the parameters $R, L$ and $K$.

To show the effects on the control, Fig. 4 represents the reference velocity (blue) and the measured one (red) and the velocity tracking. The black line represents the time instant when the parameter estimates were injected into the control. In this figure, the velocity tracking error is considerably decreased using the estimated parameters. Finally, the last figure, Fig. 5, shows the control input voltages applied to the motor. It is shown that the necessary voltages to be applied to the motor were considerably decreased, for a better velocity tracking.

The results presented on the PMSM show an application of the parameter estimation using an algebraic approach. In this application, the electrical dynamics are known, and the equations are not disturbed.

\subsection{Application 2: a magnetic bearing}

\subsubsection{Description of the experiment}

Magnetic bearings can be used for machine tool spindles to produce circular and non-circular holes with high precision. Especially in high-speed applications, magnetic bearings have won importance. Moreover, because they
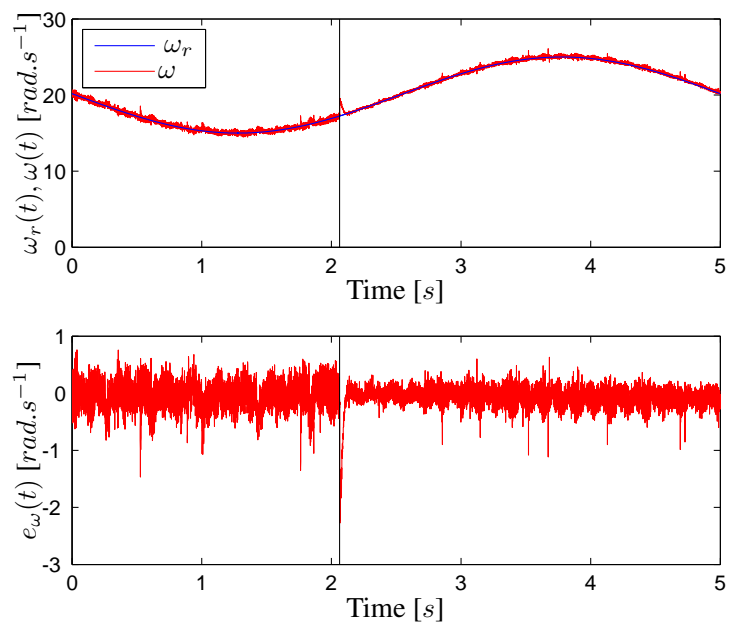

Fig. 4. Trajectory tracking evaluation of the motor velocity. 

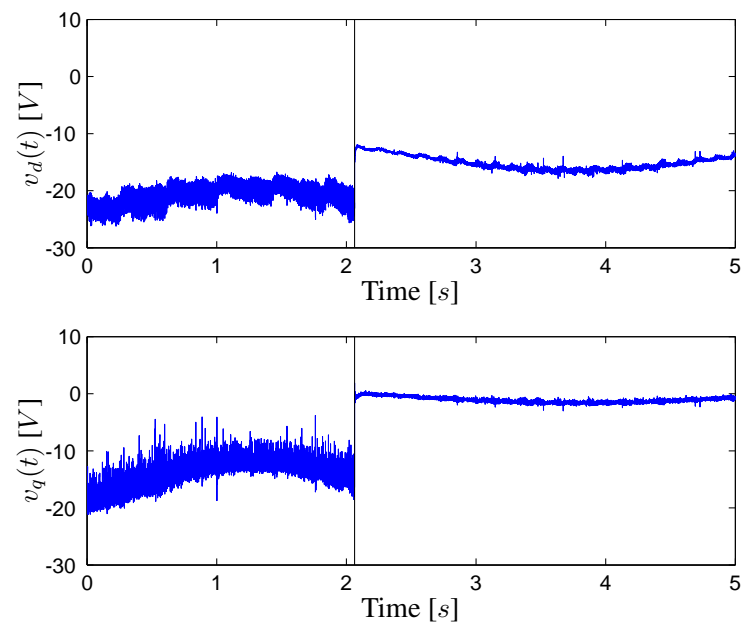

Fig. 5. Inputs voltages applied to the motor.

have a contact free suspension, they provide advantages compared to conventional bearings, such as no lubrication and no friction. This improves reliability and performance. There is a real industrial demand for such processes, which allows non-circular motion in the range of 50 micro-meters. The required precision is very important: the path tracking error must be less than 1 micrometer on circular paths and 3 micrometers on non-circular ones with a rotation speed up to $1000 \mathrm{rpm}$.

The magnetic shaft used in our laboratory consists of one electromagnetic radial bearing and two axial bearings. It is similar to the one presented in [11]. It is an inherently unstable system, where the dynamics are strongly nonlinear. The modelling simplifications lead to perturbations, which have to be considered in parameter identification. We refer the reader to $[11,17,20]$ concerning the control of magnetic shaft.

The rotor is levitated via a three-phases electromagnetic radial bearing, arranged like three coupled "horseshoe magnets" around the rotor (Fig. 6). The three generated currents are three independent control inputs. The mathematical model of the bearing is based on the assumption of a rigid body, which leads to decoupled equations for forces . Therefore, the dynamic equations can be written as follows:

$$
\begin{aligned}
m \ddot{Y} & =F_{y}, \\
m \ddot{Z} & =F_{z},
\end{aligned}
$$

where $\mathrm{Y}$ and $\mathrm{Z}$ represent the coordinates of the center of mass of the rotor in a Cartesian frame (with axes $y$ and $z$ ), which is fixed in the space at a point being considered as the center of the device. $F_{y}$ and $F_{z}$ represent the resulting

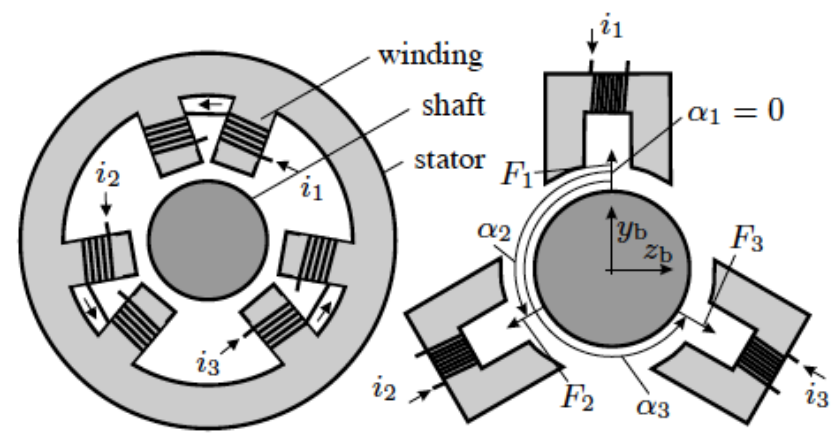

Fig. 6. Sketch of the three-phase radial bearing. 
forces applied in the directions $y$ and $z$, respectively. The rotor has a mass $m$. Gravity does not appear in these equations since the motions are in a plane perpendicular to gravity. The resulting forces in the plane $(y-z)$ are given by the superposition of the forces generated by the magnets:

$$
\left(\begin{array}{c}
F_{y} \\
F_{z}
\end{array}\right)=\left(\begin{array}{ccc}
\sin \alpha_{1} & \sin \alpha_{2} & \sin \alpha_{3} \\
\cos \alpha_{1} & \cos \alpha_{2} & \cos \alpha_{3}
\end{array}\right)\left(\begin{array}{c}
F_{1} \\
F_{2} \\
F_{3}
\end{array}\right) .
$$

The angles that appear in (31) are presented in Fig. 6. Individually, the magnetic forces can be modelled by

$$
F_{k}=\lambda_{k} \frac{i_{k}^{2}}{\left(s-\left(\begin{array}{c}
\sin \alpha_{k} \\
\cos \alpha_{k}
\end{array}\right)^{T}\left(\begin{array}{c}
Y_{b} \\
Z_{b}
\end{array}\right)\right)^{2}}, k \in\{1,2,3\}
$$

where $Y_{b}$ and $Z_{b}$ are the positions in the bearing plane, $s$ is the nominal air gap, and $\lambda_{k}$ are the parameters depending on the geometry and materials of the bearing. They will be estimated on-line.

Calculating control currents: the reference currents were obtained from the desired forces. In order to simplify the notations, it was assumed that the bearing was symmetric, i.e. $\alpha_{1}=\pi, \alpha_{2}=-\frac{\pi}{3}$ and $\alpha_{3}=\frac{\pi}{3}$. In each of the models described above, we obtained a couple of independent forces for each bearing plane. Define $F_{1}$ such that:

$$
F_{1}= \begin{cases}F_{0}, & \text { if } \quad F_{z} \geq \frac{\left|F_{y}\right|}{\sqrt{3}}, \\ F_{0}-\frac{\left|F_{y}\right|}{\sqrt{3}}+F_{z}, & \text { else. }\end{cases}
$$

With $F_{0} \geq 0$ arbitrarily chosen, the two remaining forces were obtained from the model (31):

$$
\begin{aligned}
& F_{2}=F_{1}+\frac{F_{y}}{\sqrt{3}}+F_{z}, \\
& F_{3}=F_{1}-\frac{F_{y}}{\sqrt{3}}+F_{z} .
\end{aligned}
$$

The currents were calculated from the desired magnetic forces using the relation (32).

The above equations reflect the dynamics of the unperturbed model. Model simplifications in (29)-(30) and modelling errors (e.g. due to an incorrect relationship between the input currents $i_{k}, k=y, z$ and the corresponding forces $F_{k}$, $k=y, z)$ are assumed to have a similar effect as perturbations.

The rotor rotation is also a perturbation source with harmonic components. When the rotor moves along an ellipsoid trajectory, the positions in the directions $y$ and $z$ are sinusoidal functions. A significant error in the relation between the currents and the forces can also change sinusoidally.

Therefore, the perturbations must be rejected in the $\lambda_{i}$ estimation algorithm (or must be estimated in order to be compensated for in the control).

The perturbed model can be written as:

$$
\begin{aligned}
m \ddot{Y} & =F_{y}+p_{y}(t), \\
m \ddot{Z} & =F_{z}+p_{z}(t),
\end{aligned}
$$

where $p_{k}(t), k=y, z$ represent the perturbations on each axis. Using equation (34), (35), (31) and (32), relationships of the following forms are obtained:

$$
\begin{aligned}
\ddot{Y} & =\sum_{k=1}^{3} \lambda_{k} u_{y, k}+\frac{p_{y}(t)}{m}, \\
\ddot{Z} & =\sum_{k=1}^{3} \lambda_{k} u_{z, k}+\frac{p_{z}(t)}{m},
\end{aligned}
$$


with $u_{y, k}=\frac{\sin \left(\alpha_{k}\right) i_{k}^{2}}{m\left(s-\left(\begin{array}{c}\sin \alpha_{k} \\ \cos \alpha_{k}\end{array}\right)^{T}\left(\begin{array}{c}Y_{b} \\ Z_{b}\end{array}\right)\right)^{2}}$,
and $u_{z, k}=\frac{\cos \left(\alpha_{k}\right) i_{k}^{2}}{m\left(s-\left(\begin{array}{c}\sin \alpha_{k} \\ \cos \alpha_{k}\end{array}\right)^{T}\left(\begin{array}{c}Y_{b} \\ Z_{b}\end{array}\right)\right)^{2}}$.

These two equations depend on the measured inputs and the accelerations in the plane. The parameters $\lambda_{k}$ are the parameters to be estimated from the measurements $Y$ and $Z$ despite the perturbations. The identification of magnetic bearing parameters is usually slow to be implemented and is often made off-line. In the next section, the expressions for the identification leading to an on-line, fast, and robust estimation of these parameters are developed.

\subsubsection{Estimation of $\lambda_{k}$ parameters}

The three unknown parameters $\lambda_{1}, \lambda_{2}$ and $\lambda_{3}$ can be estimated using equation (37). In order to simplify the computations, it is assumed that the perturbations are constant over a short time interval. Equation (37) is an input/output relationship of the form of equation (1) with three inputs, where $n=2, m=0$. The choice of constant perturbation implies $\kappa=1$. Using Theorem 1 for equation (37) with the three unknown parameters (i.e. $p=3$ ) leads to:

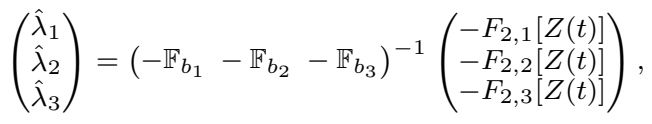

with:

$$
\mathbb{F}_{b_{*}}=\left(\begin{array}{c}
F_{0,1}\left[u_{Z, *}(t)\right] \\
F_{0,2}\left[u_{Z, *}(t)\right] \\
F_{0,3}\left[u_{Z, *}(t)\right]
\end{array}\right),
$$

where, for any function $f(t), F_{i, p}[f(t)]$ are defined in (3).

Define the vector: $\hat{\Lambda}=\left[\begin{array}{lll}\hat{\lambda}_{1} & \hat{\lambda}_{2} & \hat{\lambda}_{3}\end{array}\right]^{T}$. Equation (38) gives the expressions of the estimate of the parameters $\hat{\lambda}_{1}$, $\hat{\lambda}_{2}$ and $\hat{\lambda}_{3}$ :

$$
\Delta P_{2} \hat{\Lambda}=\Delta Q_{2},
$$

with the matrices $\Delta P_{2} \in \mathbb{R}^{3 \times 3}$ and $\Delta Q_{2} \in \mathbb{R}^{3 \times 1}$ given by:

$$
\begin{gathered}
\Delta P_{2}=\left[\begin{array}{ccc}
-F_{0,1}\left[u_{Z, 1}(t)\right] & -F_{0,1}\left[u_{Z, 2}(t)\right] & -F_{0,1}\left[u_{Z, 3}(t)\right] \\
-F_{0,2}\left[u_{Z, 1}(t)\right] & -F_{0,2}\left[u_{Z, 2}(t)\right] & -F_{0,2}\left[u_{Z, 3}(t)\right] \\
-F_{0,3}\left[u_{Z, 1}(t)\right] & -F_{0,3}\left[u_{Z, 2}(t)\right] & -F_{0,3}\left[u_{Z, 3}(t)\right]
\end{array}\right], \\
\Delta Q_{2}=\left[\begin{array}{l}
-F_{2,1}[Z(t)] \\
-F_{2,2}[Z(t)] \\
-F_{2,3}[Z(t)]
\end{array}\right]
\end{gathered}
$$

One obtains:

$$
\left[\begin{array}{l}
\hat{\lambda}_{1} \\
\hat{\lambda}_{2} \\
\hat{\lambda}_{3}
\end{array}\right]=\Delta P_{2}^{-1} \Delta Q_{2} .
$$

\subsubsection{Experimental results}

The known parameters in this experiment are the rotor mass $m=6.7 \mathrm{~kg}$ and the nominal air gap $\mathrm{s}=5.10^{-4} \mathrm{~m}$. The control is realized as a cascade, with a current controller in the inner loop and a position controller in the outer loop. The inner loop can be characterized by a control based on the electrical model of the bearing coils. The outer loop is based on the rigid body mechanical model (34)-(35). The chosen control design is a flatness based trajectory tracking control, as described in [20]. This control law is designed using an extended Luenberger observer to estimate and reject the perturbations. The reference trajectory is an elliptical one where $y^{*}=r_{Y} \cos (\omega t)$ and $z^{*}=r_{Z} \sin (\omega t)$ 


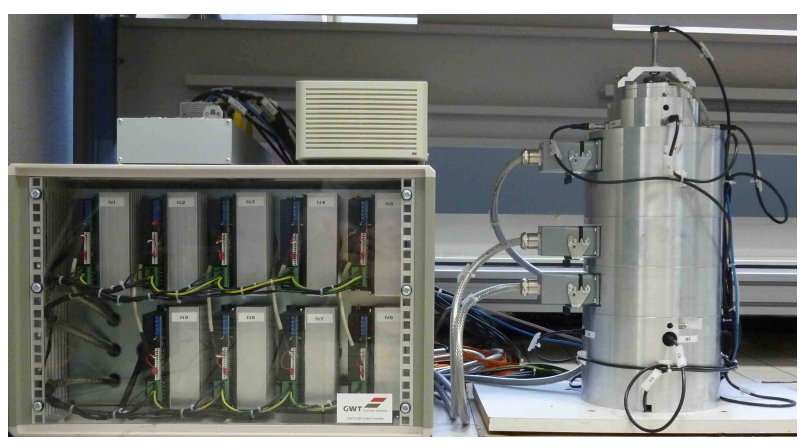

Fig. 7. Magnetic bearing test-bench.
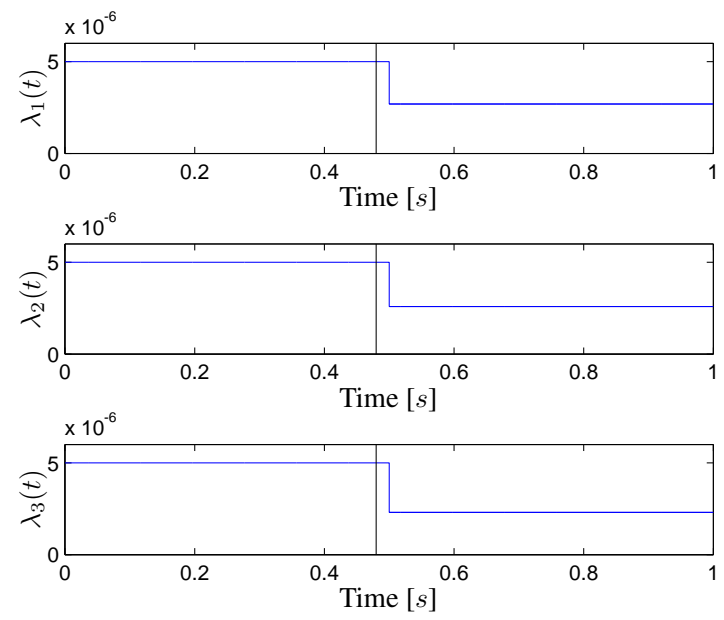

Fig. 8. Experimental estimation of the parameters $\lambda_{1}, \lambda_{2}$ and $\lambda_{3}\left[N m^{2} / A^{2}\right]$.

with $r_{Y}=30.10^{-6} \mathrm{~m}$ and $r_{Z}=70.10^{-6} \mathrm{~m}$. The shaft is rotating with an angular velocity of about $3000 \mathrm{rpm}$, i.e. $\omega=50 \cdot 2 \pi \mathrm{rad} . \mathrm{s}^{-1}$. All currents are generated by three DC brush amplifiers that serve as three independent control inputs (Fig. 11). The system's sampling period is equal to $1.10^{-4} s$. The test bench is shown Fig. 7 .

Since the dynamics of the magnetic bearing are faster than the ones of the PMSM, the window length has to be chosen shorter. Therefore, the window length was divided by 10 compared to the PMSM one. The window length was taken equal to $0.02 \mathrm{~s}$. With a sampling period of $1.10^{-4} \mathrm{~s}$, the window had 200 points, which can be implemented in real time. In order to reject the remaining harmonic perturbation with frequencies close to the rotational speed, the resulting numerator and denominator of each estimated parameter were filtered using a low-pass filter. The filter used was similar to the one presented in [32]:

$$
\hat{\lambda}_{i}(t)=\frac{F(s) n(t)}{F(s) d(t)},
$$

where $F(s)=\frac{\omega_{n}^{2}}{s^{2}+2 \zeta \omega_{n}+\omega_{n}^{2}}$, with $\zeta=0.707$ and $w_{n}=15 \mathrm{rad} / \mathrm{s}$.

Fig. 8 shows the experimental results of the identification of the parameters $\hat{\lambda}_{i}$. As mentioned before, one does not have a precise knowledge of $\lambda_{i}$. The parameters are arbitrarily set to $4 \cdot 0 \cdot 10^{-6}\left(\mathrm{Nm}^{2} / \mathrm{A}^{2}\right)$. Again, the black line represents the instant $\tau$ where the estimation started. At $t=\tau+T_{f}$, with $T_{f}=0.02 \mathrm{~s}$ here, the arbitrary values are replaced by the estimated ones. One obtains the three parameters: $\hat{\lambda}_{1}=2.53 .10^{-6} \mathrm{Nm}^{2} / A^{2}, \hat{\lambda}_{2}=2.04 .10^{-6} \mathrm{Nm}^{2} / A^{2}$ and $\hat{\lambda}_{3}=2.42 .10^{-6} \mathrm{Nm}^{2} / A^{2}$. 

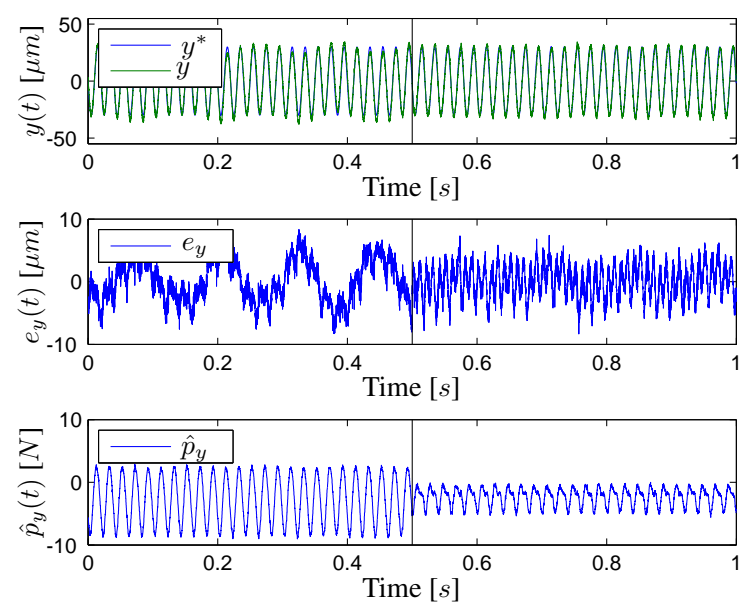

Fig. 9. Trajectory tracking evaluation on the $y$ axis.

In order to check the parameter estimation accuracy, Fig. 9 and 10 show the trajectory tracking before and after the parameter estimation along the $y$ and $z$ trajectories, respectively. A black line on the figures represents this delimitation. These figures give the reference positions (blue) and the measured ones (green), the position tracking errors and estimate of the perturbations. It can be seen on these figures that the parameter estimation does not have much as an effect on the tracking errors, although at low speed (i.e. $y$-trajectory) the error is more regular and the amplitude slightly lower. The most obvious effect is on the perturbation estimation. In fact, it is clear that the modelling is better since the amplitude of the perturbations decreases considerably when the estimated parameters are used. This observation is due to the use of an observer to estimate the perturbations. When the parameters are badly identified, the observer takes into consideration the modelling errors, hence a low tracking error. Nevertheless the currents furnished to reach this performance will be more important, as depicted in Fig. 11, where two zooms of the currents are plotted, between time $t=0.2 \mathrm{~s}$ and $0.3 \mathrm{~s}$ for the currents before identification and between $t=0.6 \mathrm{~s}$
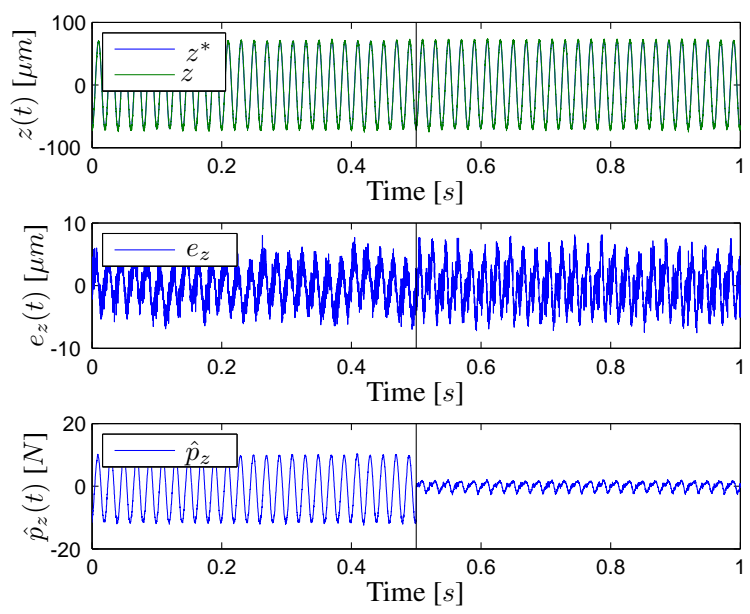

Fig. 10. Trajectory tracking evaluation on the $z$ axis. 

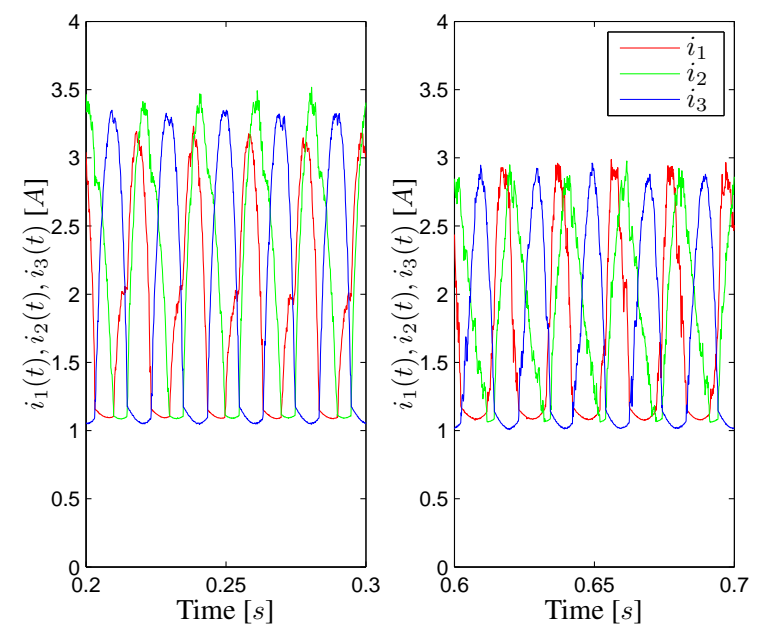

Fig. 11. Control currents before identification (left), after identification (right).

and $0.7 \mathrm{~s}$ for the currents after identification.

Finally, Fig. 12 and 13 give an idea of the shape of the hole before and after identification, where it can be seen the accuracy of the trajectory tracking is improved. One can see that the trajectory obtained with the estimated parameters presents a bias; nevertheless, the results are experimental, this bias may be due to several reasons. First, the information on the positions $y$ and $z$ are obtained by the use of eight position sensors. Each of them could be poorly calibrated and have a bias that would affect the estimation as well as the tracking. One other reason is that the control law used for these experiments is very basic. A more advanced control law could improve the tracking accuracy.

The results show that the given online estimator is able to identify parameters with very low amplitude compared to the signals and works in spite of perturbations. Moreover, the identification of three parameters simultaneously has been achieved.

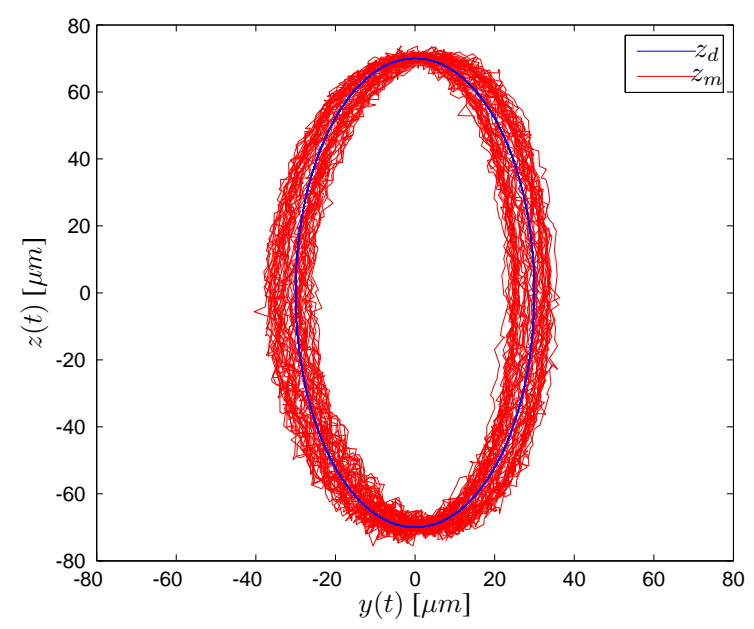

Fig. 12. Desired and measured position in the $y-z$ plane before identification. 


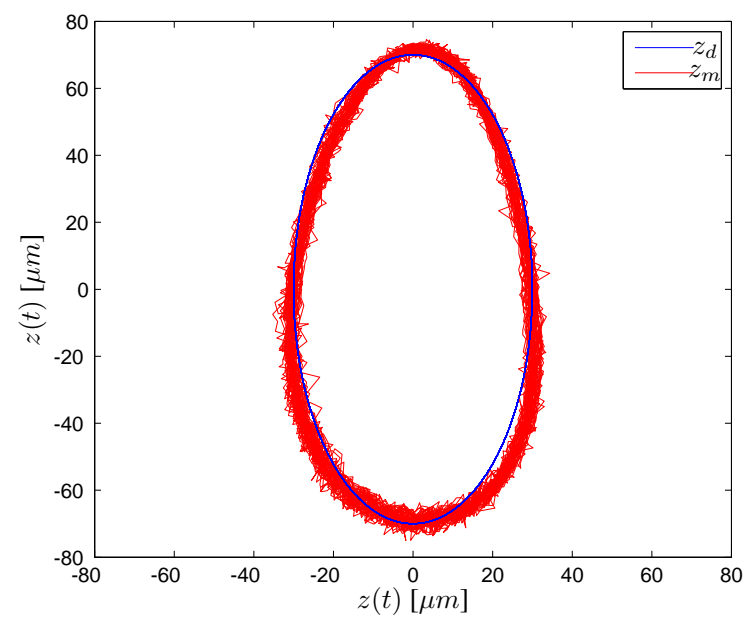

Fig. 13. Desired and measured position in the $y-z$ plane after identification.

\section{CONCLUSION}

In this article, a procedure for on-line parameter estimation using an algebraic approach has been presented. The main contributions of this article are: (i) the generalization of an existing estimator for perturbed systems, assuming that the perturbations are polynomial on a short time interval; (ii) a method to solve implementation issues in order to be able to apply this estimator for online identification. The parameter identification is obtained from the inputs and measured outputs only. It does not need to differentiate any signals. Moreover, it leads to finite time estimation and it is possible to identify several parameters simultaneously.

The proposed estimator was applied experimentally for two different processes. The estimator has shown its performance on the identification of the electrical parameters of a PMSM. Then, the algorithm was applied to identify the parameters of a magnetic bearing. The results showed that the identification is performed on a strongly nonlinear system, whereas the dynamics are affected by perturbations. Moreover, their parameters have very small amplitude compared to the signals. These two applications show that the same algorithm can easily be applied to different processes and gives good results.

The proposed estimator is robust and also fast; the estimation error is small in spite of noise and perturbations. Both experiments have shown that the estimations were obtained in less than a second. Using the on-line estimation of the parameters, the trajectories are well tracked and the inputs amplitude is lower leading to energy saving. Finally, the comparison of both identifications shows the influence of the system dynamics on the sliding window. As an improvement, it would be interesting to simultaneously identify the perturbations.

\section{REFERENCES}

1. J.-P. Barbot and T. Floquet. Iterative higher order sliding mode observer for nonlinear systems with unknown inputs. Dynamics of Continuous, Discrete and Impulsive Systems, 17(6):1019-1033, 2010.

2. F.-J. Bejarano, A. Pisano, and E. Usai. Finite-Time Converging Jump Observer for Switched Linear Systems with Unknown Inputs. Nonlinear Analysis: Hybrid Systems, 5(2):174-188, 2011.

3. L. Belkoura, T. Floquet, K. Ibn Taarit, W. Perruquetti, and Y. Orlov. Estimation problems for a class of impulsive systems. International Journal of Robust and Nonlinear Control, 21(10):1066-1079, 2010.

4. L. Belkoura, J.-P. Richard, and M. Fliess. Parameters estimation of systems with delayed and structured entries. Automatica, 45(5):1117-1125, 2009.

5. S. Bittanti and S.-M. Savaresi. On the parametrization and design of an extended Kalman filter frequency tracker. IEEE Transactions on Automatic Control, 45(9):1718-1724, September 2000.

6. A.-J. Blauch, M. Bodson, and J.-N. Chiasson. High-speed parameter estimation of stepper motors. IEEE Transactions on Control Systems Technology, 1(4):270-279, 1993. 
7. M. Bodson, J.-N. Chiasson, R.-T. Novotnak, and R.-B. Rekowski. High performance nonlinear feedback control of a permanent magnet stepper motor. IEEE Transactions on Control Systems Technology, 1(1):5-14, 1993.

8. M.-L. Corradini, G. Ippoliti, S. Longhi, D. Marchei, and G. Orlando. A Quasi-Sliding Mode Observer-based Controller for PMSM Drives. Asian Journal of Control, 15(2):380-390, 2013.

9. J. Cortes-Romero, C. Garcia-Rodriguez, A. Luviano-Juarez, and H. Sira-Ramírez. Algebraic parameter identification for induction motors. In 37th Annual Conference on IEEE Industrial Electronics Society, pages 1734-1740, Melbourne, Australia, 2011.

10. R. Delpoux, M. Bodson, and T. Floquet. Parameter estimation of permanent magnet stepper motors without position or velocity sensors. In 2012 American Control Conference, pages 1180-1185, Montréal, Québec, 2012.

11. S. Eckhardt and J. Rudolph. High Precision Synchronous Tool Path Tracking with an AMB Machine Tool Spindle. In 9th International Symposium on Magnetic Bearings, Lexington, Kentucky, USA, 2004.

12. M. Fliess, C. Join, and M. Mboup. Algebraic change-point detection. Applicable Algebra in Engineering, Communication and Computing, 21:131-143, 2010.

13. M. Fliess and H. Sira-Ramírez. An algebraic framework for linear identification. ESAIM: Control, Optimisation and Calculus of Variations, 9:151-168, January 2003.

14. M. Fliess and H. Sira-Ramírez. Closed-loop parametric identification for continuous-time linear systems via new algebraic techniques. In H Garnier \& L Wang, editor, Identification of Continuous-time Models from Sampled Data, Advances in Industrial Control, pages 362-391. Springer, 2008.

15. T. Floquet, J.-A. Twiddle, and S.-K. Spurgeon. Parameter estimation via second order sliding modes with application to thermal modelling in a high speed rotating machine. In 2006 IEEE International Conference on Industrial Technology, pages 2635-2639, Mumbai, India, 2006.

16. A. Gensior, J. Weber, J. Rudolph, and H. Guldner. Algebraic Parameter Identification and Asymptotic Estimation of the Load of a Boost Converter. IEEE Transactions on Industrial Electronics, 55(9):3352-3360, 2008.

17. T.-R. Grochmal and A. Lynch. Control of a self-bearing servomotor. IEEE Control Systems Magazine, 29(5):74-92, 2009.

18. J. Linares-Flores, H. Sira-Ramírez, E. Yescas-Mendoza, and J.-J. Vásquez-Sanjuan. A comparison between the algebraic and the reduced order observer approaches for on-line load torque estimation in a unit power factor rectifier-DC motor system. Asian Journal of Control, 14(1):45-57, 2012.

19. D.-Y. Liu, O. Gibaru, and W. Perruquetti. Error analysis of Jacobi derivative estimators for noisy signals. Numerical Algorithms, 58(1):53-83, 2011.

20. J.-V. Löwis and J. Rudolph. Flatness-based trajectory tracking control of a rotationg shaft. In 7 th International Symposium on Magnetic Bearings, pages 299-304, Zurich, Switzerland, 2000.

21. M. Mboup. Parameter estimation for signals described by differential equations. Applicable Analysis: An International Journal, 88(1):29-52, 2009.

22. M. Mboup, C. Join, and M. Fliess. Numerical differentiation with annihilators in noisy environment. Numerical Algorithms, 50:439-467, 2009.

23. R. Morales, V. Feliu, and H. Sira-Ramírez. Nonlinear Control for Magnetic Levitation Systems Based on Fast Online Algebraic Identification of the Input Gain. IEEE Transactions on Control Systems Technology, 19(4):757-771, 2011.

24. R.-H. Park. Two-reaction theory of synchronous machines generalized method of analysis-part I. Transactions of the American Institute of Electrical Engineers, 48(3):716-727, 1929.

25. A. Pisano and E. Usai. Contact force estimation and regulation in active pantographs : an algebraic observability approach. Asian Journal of Control, 13(6):761-772, 2010.

26. S. Riachy, Y. Bachalany, M. Mboup, and J.-P. Richard. Différentiation numérique multivariable I : estimateurs algébriques et structure. In Gième Conférence Internationale Francophone d'Automatique, Nancy, France, 2010.

27. J. Rudolph and F. Woittennek. An algebraic approach to parameter identification in linear infinite dimensional systems. In 16th Mediterranean Conference on Control and Automation, pages 332-337, Ajaccio, France, 2008.

28. H. Sira-Ramírez. A Passivity plus Flatness Controller for Permanent Magnet Stepper Motor. Asian Journal of Control, 2(1):1-9, 2000.

29. T. Söderström and P. Stoica. System Identification. Prentice-Hall International Editions, UK, 1989. 
30. Y. Tian, T. Floquet, and W. Perruquetti. Fast state estimation in linear time-varying systems: an algebraic approach. In 47th IEEE Conference on Decision and Control, pages 2539-2544, Mexico, Mexique, 2008.

31. Z. Tiganj, M. Mboup, C. Pouzat, and L. Belkoura. An algebraic method for eye blink artifacts detection in single channel EEG recordings. In 17th International Conference on Biomagnetism, volume 28, pages 175-178, Dubrovnik, Croatia, 2010.

32. J.-R. Trapero, H. Sira-Ramírez, and V. Feliu. An algebraic frequency estimator for a biased and noisy sinusoidal signal. Signal Processing, 87(6):1188-1201, 2007.

33. R. Ushirobira, W. Perruquetti, M. Mboup, and M. Fliess. Algebraic parameter estimation of a biased sinusoidal waveform signal from noisy data. In 16th IFAC Symposium on System Identification, Brussels, Belgique, 2012.

34. A. Verl and M. Bodson. Torque Maximization for Permanent Magnet Synchronous Motors. IEEE Transactions on Control Systems Technology, 6(6):740-745, 1998. 